\title{
Parafusos automacheantes: comparação mecânica no modelo artificial de osso e na vértebra cervical de carneiro
}

Self-tapping screws: mechanical analysis in the model of artificial bone and in the cervical sheep vertebra

\author{
Tornillos autoterrajante: comparación mecánica en el modelo \\ artificial de hueso y en la vértebra cervical de oveja
}

\author{
Patrícia Silva ${ }^{1}$ \\ Rodrigo César Rosa' \\ João Paulo Placo² \\ Antonio Carlos Shimano 3 \\ Francisco José Albuquerque de Paula ${ }^{4}$ \\ José Batista Volpon ${ }^{3}$ \\ Helton LA Defino ${ }^{3}$
}

\section{RESUMO}

Objetivo: comparar o novo parafuso travado na placa cervical (CSLP) automacheante com o parafuso convencional cortical automacheante utilizado na fixação da placa cervical anterior, por meio da análise do torque de inserção e da resistência ao arrancamento dos implantes. Métodos: 15 parafusos corticais automacheantes e 15 parafusos CSLP automacheantes foram inseridos em 15 modelos artificiais de osso (poliuretana) e 15 vértebras cervicais de carneiro. Os parâmetros estudados foram o torque de inserção e a força de arrancamento. Foram formados dois grupos experimentais, de acordo com o tipo de parafuso utilizado: Grupo I, com parafuso cortical automacheante; Grupo II, com parafuso CSLP automacheante. $\mathrm{O}$ orifício-piloto foi perfurado com 10,0 $\mathrm{mm}$ de profundidade, por meio de broca com diâmetro de 2,5

\section{ABSTRACT}

Objective: compare the new selftapping cervical spine locking plate (CSLP) screw with traditional self-tapping cortical screw used in anterior cervical locking plates in terms of insertion torque and pullout strength. Methods: 15 traditional self-tapping cortical screws and 15 new self-drilling CSLP screws were inserted into 15 models of artificial bone (polyurethane) and 15 cervical vertebrae of sheep. The studied parameters were the insertional torque and pullout strength. The following groups were created, in agreement with the type of screw used: Group I, of self-tapping cortical screw; and Group II, with self-tapping CSLP screw. The pilot hole had $10.0 \mathrm{~mm}$ in depth and was made with a 2.5 $\mathrm{mm}$ (Group I) and $3.0 \mathrm{~mm}$ (Group

\section{RESUMEN}

Objetivo: comparar el nuevo tornillo cervical spine locking plate (CSLP) autoterrajante con el convencional tornillo cortical autoterrajante utilizado en la fijación de la placa cervical anterior, por medio del análisis del torque de inserción y de la resistencia al arrancamiento de los implantes. Métodos: quince tornillos corticales autoterrajante y 15 tornillos CSLP autoterrajante fueron inseridos en 15 modelos artificiales de hueso (poliuretana) y 15 vértebras cervicales de oveja. Los parámetros estudiados fueron el torque de inserción y la fuerza de arrancamiento. Fueron formados dos grupos experimentales de acuerdo con el tipo de tornillo utilizado: Grupo I-Tornillo cortical autoterrajante; Grupo II-tornillo CSLP autoterrajante. El orificiopiloto fue perforado con $10.0 \mathrm{~mm}$ de profundidad por medio de una broca

\footnotetext{
Laboratório de Bioengenharia da Faculdade de Medicina da Universidade de São Paulo - USP - Ribeirão Preto (SP), Brasil.

Pós-graduandos da Faculdade de Medicina da Universidade de São Paulo - USP - Ribeirão Preto (SP), Brasil.

${ }^{2}$ Acadêmico do Curso de Medicina da Faculdade de Medicina da Universidade de São Paulo - USP - Ribeirão Preto (SP), Brasil.

${ }^{3}$ Professores do Departamento de Biomecânica, Medicina e Reabilitação do Aparelho Locomotor da Faculdade de Medicina da Universidade de São Paulo - USP _

Ribeirão Preto (SP), Brasil.

${ }^{4}$ Professor do Departamento de Medicina Interna da Faculdade de Medicina da Universidade de São Paulo - USP - Ribeirão Preto (SP),

Este trabalho é parte da dissertação de mestrado da primeira autora.

Trabalho realizado com apoio Fundação de Amparo à Pesquisa do Estado de São Paulo (FAPESP) e Programa CAPES-PROBRAL.
} 
mm (Grupo I) e 3,0 mm (Grupo II). O torque de inserção foi mensurado durante a implantação dos parafusos e, em seguida, foram realizados ensaios mecânicos em máquina universal de testes, para avaliar a força de arrancamento dos implantes. Resultados: os parafusos automacheantes CSLP apresentaram valores do torque de inserção e resistência ao arrancamento maiores que os parafusos corticais automacheantes. Conclusão: o desenho do parafuso CSLP influencia o seu torque de inserção e resistência ao arrancamento, sendo a avaliação desses parâmetros superiores aos parafusos corticais automacheantes.
II) drill. The insertional torque was measured and the pullout test was performed. Results: the CSLP selftapping screw presented a higher insertional torque and pullout strength compared to cortical self-tapping screws. Conclusion: the thread design of CSLP screw influenced the insertional torque and pullout resistance, and the evaluation of these parameter were superior to cortical self-tapping screw.

KEYWORDS: Spine/surgery; Bone screws; Biomechanics; Torque con diámetro de $2.5 \mathrm{~mm}$ (Grupo I) y 3.0 mm (Grupo II). El troque de inserción fue medido durante la implantación de los tornillos y en seguida fueron realizados ensayos mecánicos en máquina universal de test para evaluar la fuer$z a$ de arrancamiento de los implantes. Resultados: los tornillos autoterrajante CSLP presentaron valores del torque de inserción y resistencia al arrancamiento mayores que los tornillos corticales autoterrajante. Conclusión: el diseño del tornillo CSLP influye en su troque de inserción y resistencia al arrancamiento, siendo la evaluación de estos parámetros superiores a los tornillos corticales autoterrajante.

DESCRIPTORES: Columna/ cirugía; Tornillos óseos; Biomecánica; Torque Biomecânica; Torque

\section{INTRODUÇÃO}

A estabilidade da fixação anterior da coluna vertebral depende de vários parâmetros, como a densidade mineral óssea e a confecção do orifício-piloto ${ }^{1-4}$. A densidade mineral óssea é o principal fator que interfere na estabilidade mecânica do implante ${ }^{5,6}$, sendo esta variável não controlada pelo cirurgião.

Os parafusos foram desenvolvidos para serem inseridos nos orifícios-piloto previamente perfurados e macheados. O macheamento do orifício-piloto é utilizado para preparar o tecido ósseo adjacente para a introdução do implantes e tem sido tema controvertido no âmbito da literatura que aborda este assunto ${ }^{1-3,7}$.

O macheamento do orifício-piloto resulta em trauma adicional e aumento do tempo operatório ${ }^{3}$. Na literatura, existem relatos de que o macheamento reduziria a resistência ao arrancamento ${ }^{7-9}$. Por essa razão, mudanças nos desenhos dos parafusos foram realizadas, para eliminar a realização prévia do macheamento. Os parafusos automacheantes simplificam o procedimento cirúrgico ${ }^{1,3}$.

Contudo, mudanças nos desenhos dos parafusos podem causar repercussão no seu desempenho mecânico. $\mathrm{O}$ desempenho mecânico agudo dos implantes pode ser avaliado por meio do torque de inserção e da resistência ao arrancamento dos parafusos.

O objetivo do estudo foi comparar o novo parafuso travado na placa cervical (CSLP) automacheante ao parafuso cortical automacheante utilizado na fixação da placa cervical anterior, por meio da análise do torque de inserção e da resistência ao arrancamento dos implantes.

\section{MÉTODOS}

Foram utilizados 15 parafusos corticais automacheantes de 3,5 mm de diâmetro externo e 15 parafusos CSLP automacheante de 4,0 $\mathrm{mm}$ de diâmetro externo, ambos de titânio e com 14,0 mm de comprimento (Synthes ${ }^{\circledR}$ ) (Figura 1).

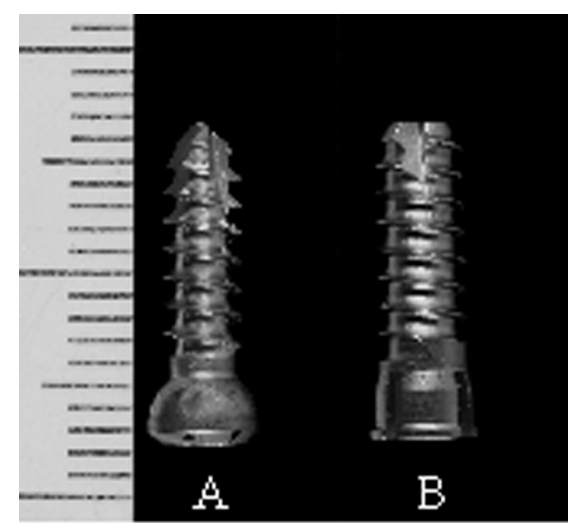

Figura 1

Parafusos utilizados no estudo. (A) Parafuso cortical automacheante; (B) Parafuso CSLP automacheante (Synthes ${ }^{\circledR}$ )

O torque de inserção e a resistência ao arrancamento foram mensurados com a inserção dos parafusos no modelo artificial de poliuretana e no corpo das vértebras cervicais de carneiro. Foram utilizados 15 corpos-de-prova de poliuretana com $40 \mathrm{~mm}$ de comprimento, $40 \mathrm{~mm}$ de largura e $40 \mathrm{~mm}$ de altura, com densidade de $0,32 \mathrm{~g} /$ $\mathrm{cm}^{3}$ (Nacional Ltda.) e 15 vértebras cervicais (C3-C6) de carneiros da raça Santa Inês deslanadados com média de 
idade de $12 \pm 3$ meses. Após sua retirada, foi removido o tecido muscular, avaliada a densidade mineral óssea pelo dual-energy X-ray absorptiometry (DEXA), utilizando o QDR system com software versão 11-2:5 (Hologic 4500 W, Waltham, MA, USA). Foram selecionadas para o estudo as vértebras com densidade mineral óssea média de $0,33 \pm 0,01 \mathrm{~g} / \mathrm{cm}^{3}\left(0,32-0,34 \mathrm{~g} / \mathrm{cm}^{3}\right)$.

Em cada corpo-de-prova, foram testados dois parafusos, cada um correspondendo a um grupo experimental. Os parafusos foram inseridos com distâncias ente si, para evitar possível interferência na mensuração do torque de inserção e da força de arrancamento.

Os grupos experimentais foram formados de acordo com o tipo de parafuso utilizado no estudo (cortical e CSLP): Grupo I, com parafuso cortical automacheante inserido após a perfuração do orifício-piloto; Grupo II, com parafuso CSLP automacheante inserido após a perfuração do orifício-piloto.

Os orifícios-piloto foram confeccionados por meio de broca com diâmetro igual ao diâmetro interno dos implantes, com a utilização de broca de 2,5 mm de diâmetro para os parafusos corticais e 3,5 mm de diâmetro para os parafusos CSLP. Ambos os orifícios foram perfurados com 10,0 mm de profundidade. Os parafusos foram inseridos no corpo anterior da vértebra cervical, simulando sua utilização clínica.

O torque de inserção dos implantes foi mensurado com microtorquímetro digital da marca MK®, modelo TI-500/ MKMT-1, 1 N.m com capacidade de resolução de 0,001 N.m; foi utilizado o software Graphic III para a análise dos dados.

Os ensaios mecânicos foram realizados utilizando máquina universal de ensaio Emic ${ }^{\circledR}$, com célula de carga com capacidade de $1.000 \mathrm{~N}$, e os dados foram analisados por meio do software Tesc 3.13.

Para a realização dos ensaios mecânicos de arrancamento, a cabeça do parafuso foi fixada à máquina de teste por conectores que permitiam movimentos multidirecionais e aplicação de carga axial de tração sem a aplicação de torque. Pré-carga de $5 \mathrm{~N}$ foi aplicada durante dez segundos para acomodação do sistema e, então, carga axial de tração foi aplicada na constante de $0,2 \mathrm{~mm} / \mathrm{min}$ até o arrancamento do implante (Figura 2).

Foram realizados 15 ensaios mecânicos e 15 medidas de torque de inserção para cada grupo experimental.
No total, 30 análises de torque e 30 testes mecânicos foram realizados.

Os resultados foram submetidos ao teste de análise de variância multifatorial (ANOVA) e pelo Paired t-test. Foi estabelecido nível de significância de $5 \%(\mathrm{p} \leq 0,05)$.

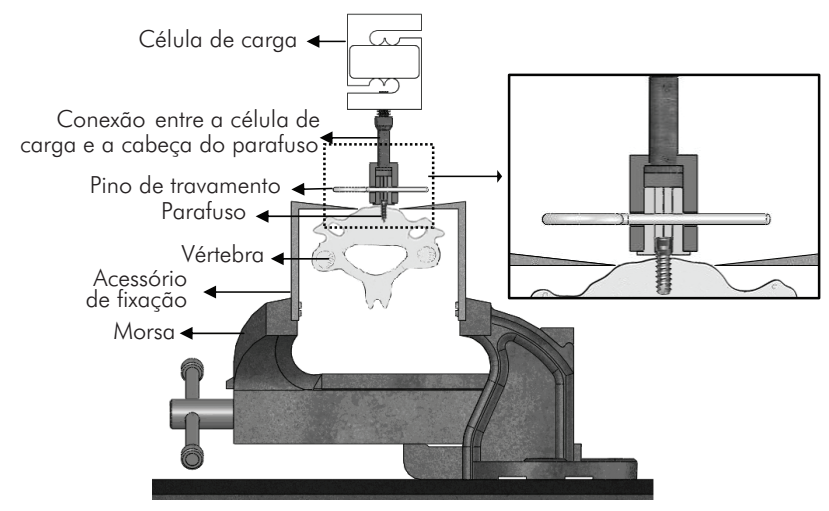

Figura 2

Desenho esquemático dos acessórios utilizados nos ensaios mecânicos. Em destaque: visão mais nítida do parafuso e dos acessórios utilizados para sua fixação.

\section{RESULTADOS}

\section{Torque de inserção}

A média máxima do torque de inserção dos parafusos implantados no modelo artificial de osso e na vértebra cervical de carneiro é representada na Tabela 1 e na Figura 3.

\begin{tabular}{|c|c|c|c|}
\hline Material & $\begin{array}{c}\text { Grupos } \\
\text { experimentais }\end{array}$ & $\begin{array}{c}\text { Torque de } \\
\text { inserção } \\
\text { (N.m) }\end{array}$ & $\begin{array}{l}\text { Valor } \\
\text { de } p\end{array}$ \\
\hline Poliuretana & $\begin{array}{l}\text { Grupo I (Cortical) } \\
\text { Grupo II (CSLP) }\end{array}$ & $\begin{array}{l}0,03 \pm 0,01 \\
0,08 \pm 0,01\end{array}$ & $\mathrm{p}<0,001$ \\
\hline Vértebras cervicais & $\begin{array}{l}\text { Grupo I (Cortical) } \\
\text { Grupo II (CSLP) }\end{array}$ & $\begin{array}{l}0,10 \pm 0,02 \\
0,16 \pm 0,02\end{array}$ & $p=0,001$ \\
\hline
\end{tabular}
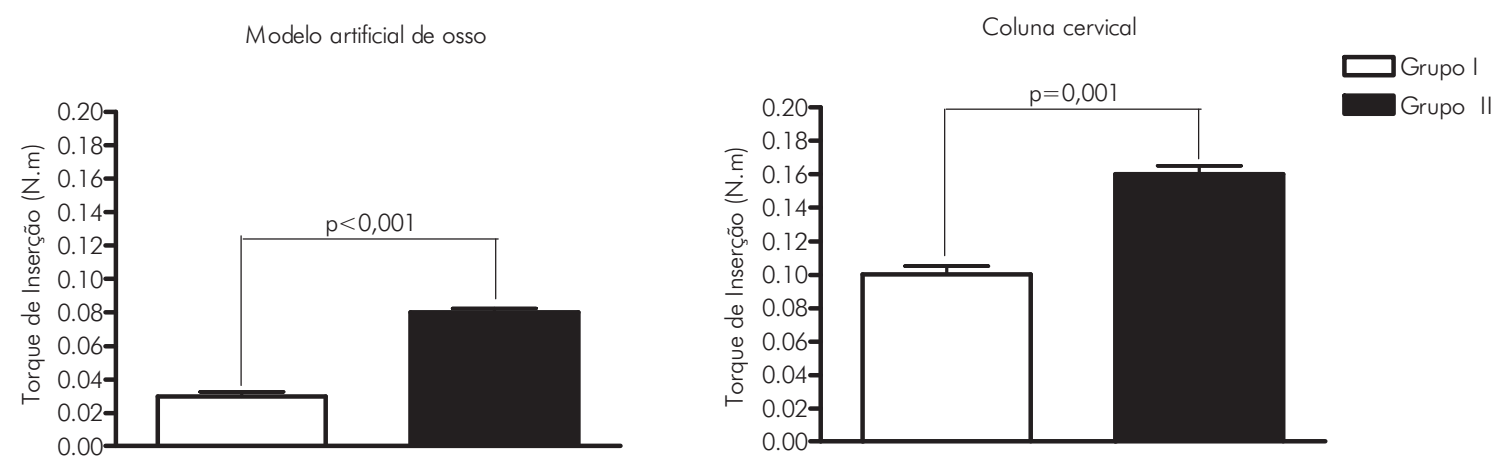

Figura 3

Comparação entre os valores médios máximos do torque de inserção nos corpos-de-prova. O nível de significância estabelecido foi $p<0,05$. 

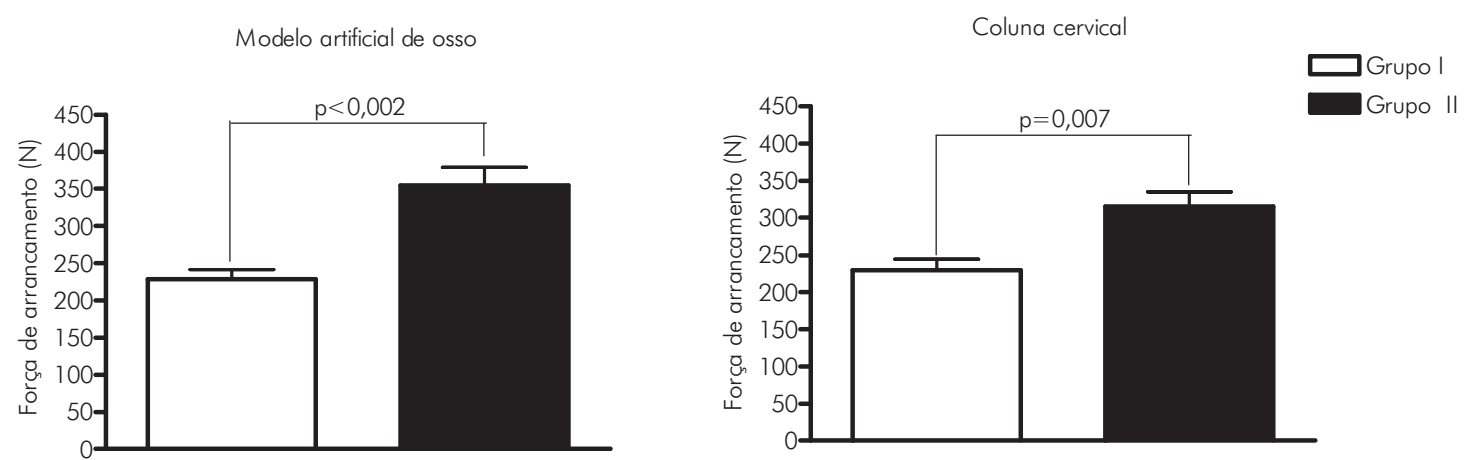

Figura 4

Comparação entre os valores médios máximos da força de arrancamento nos corpos-de-prova. $\bigcirc$ nível de significância estabelecido foi $p<0,05$

\section{Força de arrancamento}

A média máxima da força de arrancamento dos parafusos implantados na vértebra cervical de carneiro e modelo artificial de osso nos grupos de comparação está representada na Tabela 2 e Figura 4.

\section{TABELA 2 - Valores médios e desvio padrão da força média máxima de arrancamento nos corpos de prova. $O$ nível de significância estabelecido foi $p<0,05$}

\begin{tabular}{llll}
\hline Material & \multicolumn{1}{c}{$\begin{array}{c}\text { Grupos } \\
\text { experimentais }\end{array}$} & $\begin{array}{c}\text { Força de } \\
\text { arrancamento } \\
(\mathbf{n})\end{array}$ & $\begin{array}{c}\text { Valor } \\
\text { de } \mathbf{p}\end{array}$ \\
\hline Poliuretana & Grupo I (Cortical) & $228,68 \pm 50,39$ & $\mathrm{p}=0,002$ \\
& Grupo II (CSLP) & $355,12 \pm 92,20$ & \\
Vértebras & Grupo I (Cortical) & $230,15 \pm 56,02$ & $\mathrm{p}=0,007$ \\
cervicais & Grupo II (CSLP) & $314,81 \pm 77,92$ & \\
\hline
\end{tabular}

\section{DISCUSSÃO}

A inserção dos parafusos no interior das vértebras é um passo técnico de grande relevância na cirurgia da coluna vertebral, pois a fixação dos parafusos é a base para o desempenho da função biomecânica do sistema de fixação vertebral $^{10,11}$.

Os parafusos corticais de 3,5 mm automacheantes utilizados neste estudo foi o tipo inicialmente empregado para as fixações da coluna cervical ${ }^{7}$. Os novos parafusos CSLP automacheantes foram especialmente projetados para a fixação cervical, reduzindo, assim, o trauma adicional ao paciente e o tempo operatório ${ }^{1,3,12,13}$. Contudo, este estudo procurou investigar e esclarecer dúvidas referentes às características biomecânicas do novo desenho de parafuso.

O macheamento do orifício-piloto promove alterações na composição interna do osso, resultando em fraturas da matriz do tecido ósseo esponjoso, favorecendo a formação de espaços nulos e reduzindo os componentes ósseos da interface osso-implante, o que dificultaria a sua ancoragem $^{14}$. Outros estudos relatam que o macheamento, quando aplicado em osso do tipo cortical, facilitaria sua fixação, apesar da remoção do tecido ósseo ${ }^{13,15}$. No entanto, em ossos com baixa densidade ou osteoporóticos, foi observada redução de até $30 \%$ da força máxima de arrancamento $^{15}$.

Os resultados mostraram que o torque de inserção e a força de arrancamento possuem diferença significativa entre os grupos. Os parafusos CSLP automacheantes possuem melhor qualidade de fixação quando comparados aos parafusos corticais automacheantes.

$\mathrm{O}$ estudo demonstrou que a utilização do parafuso CSLP automacheante na prática clínica, além de reduzir o tempo e o número de passos cirúrgicos, proporcionou maior qualidade de fixação do sistema, quando inseridos no tecido ósseo não osteoporótico. A densidade mineral óssea das vértebras humanas normais está bem reportada na literatura e varia, em média, de 0,30 a $0,34 \mathrm{~g} / \mathrm{cm}^{3} 16,17$. As vértebras utilizadas no presente estudo, assim como os corpos-de-prova de modelo de osso artificial, possuíam densidade mineral óssea dentro dos limites de normalidade, com ausência de osteoporose ${ }^{1}$.

$\mathrm{O}$ estudo não teve como objetivo simular exatamente às condições clínica, mas fornecer medidas confiáveis referentes à ancoragem dos parafusos. Os ensaios mecânicos realizados são do tipo estático, tendo a finalidade de avaliar a resistência mecânica ao arrancamento dos implantes por meio da aplicação de carga axial ao longo do seu eixo e permitem comparação simples e segura ${ }^{13}$.

A resistência ao arrancamento dos parafusos é um fenômeno complexo e multifatorial. O novo desenho dos parafusos CSLP automacheantes elimina a realização prévia do macheamento do orifício-piloto, reduzindo uma das variáveis que influenciam na qualidade do sistema de fixação

\section{CONCLUSÃO}

Os parafusos CSLP automacheantes apresentam maiores valores médios do torque de inserção e resistência ao arrancamento quando comparados aos parafusos corticais automacheantes. 


\section{REFERÊNCIAS}

1. Conrad BP, Cordista AG, Horodyski M, Rechtine GR. Biomechanical evaluation of the pullout strength of cervical screws. J Spinal Disord Tech. 2005;18(6):506-10.

2. Defino HLA, Wich CRH, Shimano AC, Kandziora F. Influência do diâmetro do orifício piloto na resistência ao arrancamento dos parafusos do corpo vertebral. Acta Ortop Bras. 2007;15(2):76-9.

3. Hitchon PW, Brenton MD, Coppes JK, From AM, Torner JC. Factors affecting the pullout strength of self-drilling and self-tapping anterior cervical screws. Spine (Phila Pa 1976). 2003;28(1):913.

4. Ryken TC, Clausen JD, Traynelis VC, Goel VK. Biomechanical analysis of bone mineral density, insertion technique, screw torque, and holding strength of anterior cervical plate screws. J Neurosurg. 1995;83(2):3259.

5. Coe JD, Warden KE, Herzig MA, McAfee PC. Influence of bone mineral density on the fixation of thoracolumbar implants. A comparative study of transpedicular screws, laminar hooks, and spinous process wires. Spine (Phila Pa 1976). 1990;15(9):902-7.

6. Wittenberg RH, Shea M, Swartz DE, Lee KS, White AA 3rd, Hayes WC. Importance of bone mineral density in instrumented spine fusions. Spine (Phila Pa 1976). 1991;16(6):647-52.
7. Carmouche JJ, Molinari RW, Gerlinger T, Devine J, Patience T. Effects of pilot hole preparation technique on pedicle screw fixation in different regions of the osteoporotic thoracic and lumbar spine. J Neurosurg Spine. 2005;3(5):364-70.

8. Kwok AW, Finkelstein JA, Woodside T, Hearn TC, Hu RW. Insertional torque and pull-out strengths of conical and cylindrical pedicle screws in cadaveric bone. Spine (Phila Pa 1976). 1996;21(21):2429-34.

9. Pfeiffer FM, Abernathie DL, Smith DE. A comparison of pullout strength for pedicle screws of different designs: a study using tapped and untapped pilot holes. Spine (Phila Pa 1976). 2006;31(23):E867-70.

10. Benzel EC. Implant-bone interfaces. In: Benzel EC, editors. Biomechanics of spinal stabilization. New York: Thieme; 2001. p. 155-70.

11. Defino HL, Vendrame JR. Role of cortical and cancellous bone of the vertebral pedicle in implant fixation. Eur Spine J. 2001;10(4):325-33.

12.Pfeiffer M, Gilbertson LG, Goel VK, Griss P, Keller JC, Ryken TC, et al. Effect of specimen fixation method on pullout tests of pedicle screws. Spine (Phila Pa 1976). 1996;21(9):1037-44.

13.Ronderos JF, Jacobowitz R, Sonntag VK, Crawford NR, Dickman CA. Comparative pull-out strength of tapped and untapped pilot holes for bicortical anterior cervical screws. Spine (Phila Pa 1976). 1997;22(2):167-70.
14.Oktenoğlu BT, Ferrara LA, Andalkar N, Ozer AF, Sarioğlu AC, Benzel EC. Effects of hole preparation on screw pullout resistance and insertional torque: a biomechanical study. J Neurosurg. 2001;94(1Suppl):91-6.

15.Orozco DR, Llovet TJ. Osteosínteses en las fracturas de raquis cervical. Rev Ortop Traumatol. 1970;14:285-8.

16.Hsu CC, Chao CK, Wang JL, Hou SM, Tsai YT, Lin J. Increase of pullout strength of spinal pedicle screws with conical core: biomechanical tests and finite element analyses. J Orthop Res. 2005;23(4):788-94.

17.Kandziora F, Pflugmacher R, Scholz M, Schnake K, Lucke M, Schroder R, et al. Comparison between sheep and human cervical spines: an anatomic, radiographic, bone mineral density, and biomechanical study. Spine (Phila Pa 1976). 2001;26(9):1028-37.

"Comment in: Spine (Phila Pa 1976). 2001 Dec 1;26(23):2640-1.

\begin{tabular}{l} 
Correspondência \\
Helton LA Defino \\
Avenida Bandeirantes, 3.900, $11^{\circ}$ \\
andar - Monte Alegre - \\
CEP: $114048-900$ - Ribeirão Preto (SP), \\
Brasil \\
Tel: (16) 3633-0336 \\
E-mail: hladefin@fmrp.usp.br \\
\hline
\end{tabular}

